\title{
A CONVERGENCE QUESTION IN $H^{p}$
}

\section{STEPHEN SCHEINBERG ${ }^{1}$}

Abstract. Let $\phi \in H^{p}$ (unit disc), $0<p<\infty$. and let $\phi_{r}(z)=\phi(r z)$, $r<1$. If $\phi$ contains a nontrivial inner factor, it is known that $\phi / \phi_{r}$ is unbounded in $H^{p}$-norm. We prove that if $\phi$ is analytic on the closed disc and has no zeros on the open disc, then $\phi / \phi_{r} \rightarrow 1$ in $H^{p}$, as $r \rightarrow 1$. The same conclusion follows if $1 / \phi \in H^{\infty}$. We construct an outer function $\phi$ which is continuous on the closed disc, analytic for $z \neq 1$, and such that $\phi / \phi_{r}$ is unbounded in every $H^{p}$.

The following question, originating with G. Lumer, I believe, was put to me by L. Zalcman: if $\phi \in H^{2}$ and is outer, does it follow that $\phi(z) / \phi(r z) \rightarrow 1$ in $H^{2}$ as $r \rightarrow 1^{-}$? This note provides the following answers to this question. ${ }^{2}$ It will be clear that the same results hold in $H^{p}$, for $0<p<\infty$.

THEOREM. (a) If it is also true that $|\phi| \geqq \delta>0$ on $\{|z|<1\}$, then the answer is "yes".

(b) If $\phi$ is actually analytic on $\{|z| \leqq 1\}$, then the answer is "yes."

(c) There is a $\phi$ which is continuous on $\{|z| \leqq 1\}$, is analytic on $\{|z| \leqq 1, z \neq 1\}$, is outer and has

$$
\limsup _{r \rightarrow 1} \int\left|\frac{\phi\left(e^{i \theta}\right)}{\phi\left(r e^{i \theta}\right)}\right|^{\epsilon} d \theta=+\infty,
$$

for any $\epsilon>0$. Hence, in particular, $\phi\left(e^{i \theta}\right) / \phi\left(r e^{i \theta}\right) \nrightarrow 1$ in $L^{2}$.

The positive results (a) and (b) and the negative result (c) provide natural limitations on each other and are in a certain sense best possible. The basic notions and facts of $H^{p}$-theory can be found in Hoffman's book. ${ }^{3}$ The proofs of (a), (b), and (c) involve computations;

Received by the editors May 21, 1970.

AMS 1969 subject classifications. Primary 3067, 3085.

Key words and phrases. Inner function, outer function.

${ }^{1}$ Preparation of this paper was supported in part by NSF GP 11911.

2 As the referee points out, part (c) of the Theorem answers in the negative the following natural question in prediction theory. Consider the classical prediction problem for one-parameter stationary processes and obtain the formal expression for the predictor from the Taylor coefficients (at 0 ) of the inverse of the generating function. Is this formal expression necessarily Abel-summable?

${ }^{3}$ Kenneth Hoffman, Banach spaces of analytic functions, Prentice-Hall Series in Modern Analysis, Prentice-Hall, Englewood Cliffs, N. J., 1962. MR 24 \#A2844. 
(a) and (b) are straightforward; (c) involves manipulations of the Poisson kernel, and the example is motivated by the inner function $\exp [(z+1) /(z-1)]$, for which the convergence in question is well known to fail. This last fact is the reason for the hypothesis that $\phi$ be outer.

REMARK.

$$
0 \leqq\left|1-\frac{\phi\left(e^{i \theta}\right)}{\phi\left(r e^{i \theta}\right)}\right|^{2}=1-2 \operatorname{Re} \frac{\phi\left(e^{i \theta}\right)}{\phi\left(r e^{i \theta}\right)}+\left|\frac{\phi\left(e^{i \theta}\right)}{\phi\left(r e^{i \theta}\right)}\right|^{2} .
$$

$\operatorname{Re}\left(\phi\left(e^{i \theta}\right) / \phi\left(r e^{i \theta}\right)\right)$ are the boundary values of a harmonic function which is 1 at the origin. Therefore,

$$
0 \leqq \int\left|1-\frac{\phi\left(e^{i \theta}\right)}{\phi\left(r e^{i \theta}\right)}\right|^{2} \frac{d \theta}{2 \pi}=1-2+\int\left|\frac{\phi\left(e^{i \theta}\right)}{\phi\left(r e^{i \theta}\right)}\right|^{2} \frac{d \theta}{2 \pi} .
$$

Thus, the original question is equivalent to

$$
\text { "does } \int_{-\pi}^{\pi}\left|\frac{\phi\left(e^{i \theta}\right)}{\phi\left(r e^{i \theta}\right)}\right|^{2} \frac{d \theta}{2 \pi} \rightarrow 1 \text { ?" }
$$

When " 2 " is replaced by " $p$ ", the same equivalence holds. It is a standard fact of Lebesgue theory that if $f_{r} \rightarrow f$, a.e., and $\int\left|f_{r}\right|^{p} \rightarrow \int|f|^{p}$, then $f_{r} \rightarrow f$ in $L^{p}$.

PROoFs. (a) If $|\phi| \geqq \delta>0$, then $1 / \phi \in H^{\infty}$ and since $\phi\left(r e^{i \theta}\right) \rightarrow \phi\left(e^{i \theta}\right)$, a.e., we have $\phi\left(e^{i \theta}\right) / \phi\left(r e^{i \theta}\right) \rightarrow 1$, a.e., and dominated by the $L^{2}$ function const $\left|\phi\left(e^{i \theta}\right)\right|$. The same proof works in $H^{p}$.

(b) If $\phi$ is analytic on $|z| \leqq 1$ and is outer, it has the form $\phi(z)$ $=\left(z-e^{i \theta_{1}}\right)^{n_{1}} \cdots\left(z-e^{i \theta_{q}}\right)_{n_{q}} \psi(z)$, where $\theta_{1}, \cdots$ are distinct in $0 \leqq \theta$ $<2 \pi$ and both $\psi$ and $1 / \psi$ are analytic for $|z| \leqq 1$.

It is sufficient to show $\left|\left(1-e^{i \theta}\right) /\left(1-r e^{i \theta}\right)\right|^{m} \rightarrow 1$ in $L^{2}(d \theta)$, for each $m \geqq 1$. For if this is the case, then $\left|\phi\left(e^{i \theta}\right) / \phi\left(r e^{i \theta}\right)\right|=$ product of terms, each of which converges to 1 in $L^{2}$ and pointwise, a.e. On any small interval of $\theta$ 's, at most one term is unbounded as $r \rightarrow 1$. Thus, we will be done by the Remark above. I thank D. Neu for the following argument, which considerably simplifies the proof. Since

$$
\left|\frac{1-e^{i \theta}}{1-r e^{i \theta}}\right| \leqq 2 \text { and } \frac{1-e^{i \theta}}{1-r e^{i \theta}} \rightarrow 1 \text {, a.e., }
$$

the Lebesgue bounded convergence theorem gives the desired result.

(c) The motivation is the inner function $e^{(z+1) /(z-1)}$ (as mentioned), and the example is constructed and verified from very elementary properties of the Poisson kernel 


$$
P(r, \theta)=\frac{1-r^{2}}{1-2 r \cos \theta+r^{2}}=\operatorname{Re}\left(\frac{1+r e^{i \theta}}{1-r e^{i \theta}}\right) .
$$

This well-known fact is the basis for the construction:

$$
\int_{-\delta}^{\delta} P(r, \theta)^{1+\epsilon} d \theta \rightarrow \infty \quad \text { as } r \rightarrow 1^{-}, \quad \text { for any } \epsilon>0, \delta>0 .
$$

$\left(\right.$ Let $f(\theta)=n^{(\epsilon /(1+\epsilon))}$ for $-1 / 2 n<\theta<1 / 2 n$ and 0 otherwise; then

$$
\int_{-\delta}^{\delta} P(r, \theta) f(\theta) d \theta \rightarrow n^{(\epsilon /(1+\epsilon))} \quad \text { as } r \rightarrow 1^{-} \text {. }
$$

However,

$$
\int P f \leqq\left(\int P^{1+\epsilon}\right)^{(1 /(1+\epsilon))}\left(\int f^{((1+\epsilon) / \epsilon)}\right)^{(\epsilon /(1+\epsilon))}=\left(\int P^{1+\epsilon}\right)^{(1 /(1+\epsilon))} .
$$

Thus

$$
n^{(\epsilon /(1+\epsilon))} \leqq \lim \inf \left(\int P^{1+\epsilon}\right)^{(1 /(1+\epsilon))} \text { or } n^{\epsilon} \leqq \lim \inf \left(\int P^{1+\epsilon}\right) .
$$

This, for all $n$, implies $\lim \inf \left(\int P^{1+\epsilon}\right)=+\infty$, as desired.)

In particular, $\int_{-\delta}^{\delta}[P(r, \theta)]^{2} d \theta \rightarrow \infty$ and hence $\int_{-\delta}^{\delta} \exp \epsilon P(r, \theta) d \theta$ $\rightarrow \infty$ as $r \rightarrow 1^{-}$, for any $\epsilon>0, \delta>0$.

The function $\phi$ will be $\phi(z)=\prod_{n=1}^{\infty} \exp \epsilon_{n}\left(\left(r_{n} z+1\right) /\left(r_{n} z-1\right)\right)$ where $\epsilon_{n}>0$ and $\sum \epsilon_{n}=1$ and $r_{n} \rightarrow 1^{-}$(to be chosen).

Observe that any such $\phi$ is analytic on $\{|z| \leqq 1, z \neq 1\}$, continuous on $\{|z| \leqq 1\}$ and outer. Indeed, since $\left(r_{n} z+1\right) /\left(r_{n} z-1\right) \rightarrow(z+1) /(z-1)$ uniformly (as $n \rightarrow \infty)$ for all $z$ with $|z-1| \geqq \delta>0$ (for any $\delta$ ) and $\sum \epsilon_{n}<\infty, \phi$ is analytic on the complement of $\left\{1 / r_{1}, 1 / r_{2}, \cdots, 1\right\}$. To prove continuity at $z=1$, first observe that $\log \left|\phi\left(r e^{i \theta}\right)\right|=$ $-\sum \epsilon_{n} P\left(r r_{n}, \theta\right)$, so that $\log |\phi|$ is the Poisson integral of the function $-\sum \epsilon_{n} P\left(r_{n}, \theta\right)$. This proves $\phi$ is outer. We will be finished if we show $\sum \epsilon_{n} P\left(r_{n}, \theta\right)$ is continuous from $-\pi \leqq \theta \leqq \pi$ to $[0, \infty]$. Each $P\left(r_{n}, \theta\right)$ is even and is monotonic on either side of $\theta=0$; the same holds for the sum, since $\epsilon_{n}>0$. Since $\int \sum d \theta / 2 \pi=1, \sum$ cannot be identically $+\infty$ in any interval; so $\sum$ converges at each $\theta$, except possibly $\theta=0$. Monotonicity gives uniform convergence for $|\theta| \geqq \delta>0$, any $\delta$. Monotonicity and evenness imply that as $\theta \rightarrow 0, \sum$ approaches either some finite limit or $+\infty$, and it is easy to see that this limit is $\sum(0)$. In any case, the product converges to $\phi$ uniformly on $|z| \leqq 1$. 
Now select any $\epsilon_{n}>0, \sum \epsilon_{n}=1$ (say, $\epsilon_{n}=2^{-n}$ ). Inductively, we will select $\rho_{1}<r_{1}<\rho_{2}<r_{2}<\cdots$ and $I_{1} \supseteq I_{2} \supseteq \cdots$ open intervals containing 0 so that $\rho_{n} \rightarrow 1, I_{n} \downarrow\{0\}$ and the $\phi(z)$ defined above for $\left\{\left(\epsilon_{n}, r_{n}\right)\right\}$ has

$$
\int_{-\pi}^{\pi}\left|\frac{\phi\left(e^{i \theta}\right)}{\phi\left(\rho_{n} e^{i \theta}\right)}\right|^{(1 / n)} \frac{d \theta}{2 \pi}>n .
$$

(The purpose of $I_{n}$ will become apparent.)

Inductively, we may assume $\rho_{k}, r_{k}, I_{k}$ are defined for all $k<n$. (The case $n=1$ is vacuous.) Let $a_{n}=\min _{\theta} \exp -\sum_{k<n} \epsilon_{k} P\left(r_{k}, \theta\right)$. Then $0<a_{n} \leqq 1\left(a_{1}=1\right.$ since the empty sum $\left.=0\right)$. For convenience, let $I_{0}$ $=(-\pi, \pi)$.

Since $\int \exp \epsilon P(r, \theta) d \theta / 2 \pi \rightarrow \infty$ as $r \rightarrow 1$, no matter what $\epsilon>0$ is, let $\rho_{n}$ be chosen between $r_{n-1}$ and 1 and so close to 1 that

$$
\int_{I_{n-1}} \exp \frac{1}{n} \epsilon_{n} P\left(\rho_{n}, \theta\right) \frac{d \theta}{2 \pi}>\frac{(n+1) e}{a_{n}} .
$$

Now choose $I_{n} \subseteq I_{n-1}$ so small that $\int_{I_{n-1}-I_{n}}$ is still $>\left(n+\frac{1}{2}\right) e / a_{n}$. Now we can choose $r_{n}$ between $\rho_{n}$ and 1 and so close to 1 that $P\left(r_{n}, \theta\right)<1$ outside $I_{n}$, since $P(r, \theta) \rightarrow 0$ uniformly outside $I_{n}$, and

$$
\int_{I_{n-1}-I_{n}} \exp \frac{1}{n} \epsilon_{n} P\left(r_{n} \rho_{n}, \theta\right) \frac{d \theta}{2 \pi}>\frac{n e}{a_{n}},
$$

since $P(r \rho, \theta) \rightarrow P(\rho, \theta)$ uniformly as $r \rightarrow 1$.

This inductive step defines $\rho_{n}, r_{n}, I_{n}$ for all $n \geqq 1$ and we put $\phi(z)$ $=\prod_{1}^{\infty} \exp \epsilon_{n}\left(r_{n} z+1\right) /\left(r_{n} z-1\right)$. It remains to show

$$
\begin{gathered}
\int_{-\pi}^{\pi}\left|\frac{\phi\left(e^{i \theta}\right)}{\phi\left(\rho_{n} e^{i \theta}\right)}\right|^{(1 / n)} \frac{d \theta}{2 \pi} \geqq n . \\
\left|\frac{\phi\left(e^{i \theta}\right)}{\phi\left(\rho_{n} e^{i \theta}\right)}\right|^{(1 / n)}=\exp \frac{1}{n} \sum_{k=1}^{\infty} \epsilon_{k}\left[P\left(r_{k} \rho_{k}, \theta\right)-P\left(r_{k}, \theta\right)\right] \\
\geqq \exp \left(\frac{1}{n} \sum_{1}^{n-1}\right) \cdot \exp \left(\frac{1}{n} \epsilon_{n} P\left(r_{n} \rho_{n}, \theta\right)\right) \cdot \exp \left(-\frac{1}{n} \sum_{n}^{\infty} \epsilon_{k} P\left(r_{k}, \theta\right)\right),
\end{gathered}
$$

discarding all the positive exponents $P\left(r_{k} \rho_{k}, \theta\right)$ for $k \geqq n+1$. Examining these three factors on $I_{n-1}-I_{n}$ we find: The first $\geqq a_{n}^{(1 / n)} \geqq a_{n}$, by discarding the $P\left(r_{k} \rho_{n}, \theta\right)$ from the exponent. The third $\geqq \exp \left(-(1 / n) \sum_{n}^{\infty} \epsilon_{k} \cdot 1\right)>\exp (-1 / n) \geqq e^{-1}$, since $I_{k} \supset I_{k+1} \supset \cdots$ and $P\left(r_{k}, \theta\right)<1$ outside $I_{k}$. The second has $\int_{I_{n-1}-I_{n}} \cdots d \theta / 2 \pi>n e / a_{n}$ by choice. Therefore, 
124

STEPHEN SCHEINBERG

$$
\int_{-\pi}^{\pi}\left|\frac{\phi\left(e^{i \theta}\right)}{\phi\left(\rho_{n} e^{i \theta}\right)}\right|^{(1 / n)} \frac{d \theta}{2 \pi}>\int_{I_{n-1}-I_{n}} \cdots \frac{d \theta}{2 \pi}>a_{n} \cdot \frac{n e}{a_{n}} \cdot e^{-1}=n,
$$

as claimed.

It is clear that

$$
\lim _{n} \int\left|\frac{\phi\left(e^{i \theta}\right)}{\phi\left(\rho_{n} e^{i \theta}\right)}\right|^{\bullet} d \theta=+\infty
$$

for any $\epsilon>0$.

Stanford University, Stanford, California 94305 